\title{
Continuous-flow left ventricular assist devices and bleeding events: Is there a biological role for decreasing pump speed?
}

\author{
Shahab A. Akhter, MD
}

\footnotetext{
From the Division of Cardiothoracic Surgery, University of Wisconsin School of Medicine and Public Health, Madison, Wis.

Disclosures: Author has nothing to disclose with regard to commercial support

Received for publication Feb 10, 2016; accepted for publication Feb 10, 2016; available ahead of print March 11, 2016.

Address for reprints: Shahab A. Akhter, MD, Division of Cardiothoracic Surgery, University of Wisconsin School of Medicine and Public Health, 600 Highland Avenue, CSC H4/340, Madison, WI 53792 (E-mail: akhter@ surgery.wisc.edu).

J Thorac Cardiovasc Surg 2016;151:1755-6

$0022-5223 / \$ 36.00$

Copyright (c) 2016 by The American Association for Thoracic Surgery

http://dx.doi.org/10.1016/j.jtcvs.2016.02.027
}

This study by Kang and co-authors ${ }^{1}$ investigates the effect of decreasing HeartMate II (Thoratec Corporation, Pleasanton, Calif) left ventricular assist device (LVAD; Figure 1) speed on von Willebrand factor (vWF) degradation. The primary clinical problem being addressed is the relatively high incidence of bleeding associated with continuousflow LVADs and evaluating whether a lower speed may decrease shear force and subsequent vWF degradation. The authors measure vWF degradation products in blood samples taken from patients $(\mathrm{n}=10)$ pre- and post-LVAD implant and use a mock flow loop with human donor blood and a HeartMate II LVAD in the circuit at 11,400, 10,000, and $8400 \mathrm{rpm}$ for 12 hours. At 7 days post-LVAD implant, there is a 2-fold increase in vWF degradation products compared with preoperative baseline values. Of note, 6 of the 10 patients experienced significant bleeding episodes requiring hospitalization (4 gastrointestinal [GI] and 2 intracranial bleeds) at a mean speed of $9040 \mathrm{rpm}$. Likewise, in the mock flow loop, there was a significant increase in vWF degradation products at 6 and 12 hours at all 3 speeds with supraphysiologic shear stress even at $8400 \mathrm{rpm}$. The authors conclude that decreasing continuous-flow LVAD speed in this mock flow loop has no impact on vWF degradation; therefore, this does not seem to be a valid clinical strategy in the management of postimplant bleeding.

The study addresses the significant and common clinical complication of bleeding (mucosal, intracranial) associated with $30 \%$ to $75 \%$ of patients with continuous-flow devices in different published series as referenced in this article. ${ }^{1}$ An acquired vWF syndrome develops that likely contributes to the bleeding episodes in these patients. It has been proposed that decreasing the LVAD speed and increasing native pulsatility may be therapeutic. On the basis of the data from this study, ${ }^{1}$ it would not be effective solely on the basis of vWF degradation, which is not affected going from 11,400 to $8400 \mathrm{rpm}$. There has been some anecdotal evidence suggesting that decreasing LVAD speed does reduce bleeding, and the authors propose that this may be tion, Pleasanton, Calif).

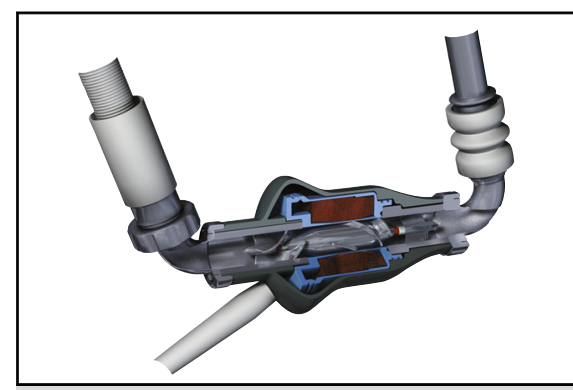

HeartMate II left ventricular assist device (Thoratec, Pleasanton, Calif).

\section{Central Message}

Decreasing HeartMate II (Thoratec Corp, Pleasanton, Calif) LVAD speed within the clinical operational range in a mock circulatory loop does not decrease vWF degradation and is unlikely to affect clinical bleeding events.

See Article page 1747.

due to more blood flowing through the native left ventricular outflow tract with less flow through the LVAD being subjected to high shear stress. This is clearly different from flow through the mock circulatory flow loop, where there is no alternate flow pathway. This is a limitation of the ex vivo design of the study relative to the actual physiology in patients post-LVAD implant.

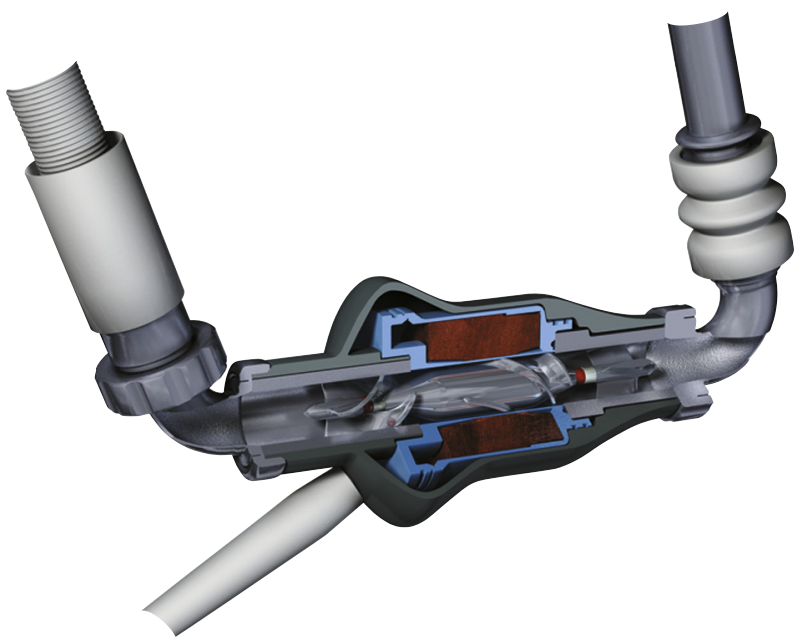

FIGURE 1. HeartMate II left ventricular assist device (Thoratec Corpora- 
The fine balance between bleeding and thrombosis in the patient population with continuous-flow LVADs remains a significant clinical challenge. It has recently been demonstrated that GI bleeding represents the most frequent cause of hospital readmission and is associated with substantial cost for evaluation and therapy. ${ }^{2}$ Many of these patients have recurrent admissions for bleeding, which significantly affects quality of life and can increase sensitization, which is particularly important in the bridgeto-transplant population. A recent study demonstrated that GI bleeding during continuous-flow LVAD support also is associated with significantly lower rates of cardiac transplantation. ${ }^{3}$

The current study provides strong evidence that decreasing the HeartMate II LVAD speed to as low as $8400 \mathrm{rpm}$ does not have any significant impact on degradation of vWF, but it is in the setting of the mock circulatory flow loop where there is no other path for blood flow. This would be a difficult study to perform clinically because lower speeds are associated with increased risk of thrombosis. There is potential that the inci- dence of bleeding associated with continuous-flow LVADs will decrease with the next-generation HeartMate III, which is currently being evaluated by a large multicenter clinical trial in the United States and already has received CE Mark approval in Europe. Novel aspects of the design of this magnetically levitated centrifugal flow pump include greater hemocompatibility and the generation of a pulse, which may have an impact on postimplant bleeding. This would represent a major advance in clinical outcomes and quality of life for the LVAD recipient.

\section{References}

1. Kang J, Zhang DM, Restle DJ, Kallel F, Acker MA, Atluri P, et al. Reduced continuous-flow left ventricular assist device speed does not decrease von Willebrand factor degradation. J Thorac Cardiovasc Surg. 2016;151:1747-54.e1.

2. Akhter SA, Badami A, Murray M, Kohmoto T, Lozonschi L, Osaki S, et al. Hospital readmissions after continuous-flow left ventricular assist device implantation: incidence, causes, and cost analysis. Ann Thorac Surg. 2015;100: 884-9.

3. Holley CT, Harvey L, Roy SS, Cogswell R, Eckman P, Liao K, et al. Gastrointestinal bleeding during continuous-flow left ventricular assist device support is associated with lower rates of cardiac transplantation. ASAIO J. 2015;61:635-9. 